\author{
120017753_AGB31_03_R1_121902 \\ COMMUNICATIONS IN ALGEBRA ${ }^{\circledR}$ \\ Vol. 31, No. 3, pp. 1085-1111, 2003
}

\title{
Trace Properties and Pullbacks
}

10

11

12

13

14

15

16

17

18

19

20

21

22

23

24

25

26

27

28

29

30

31

32

33

34

35

36

37

38

39

40

41

42

*Correspondence: Thomas G. Lucas,, Department of Mathematics, University of North Carolina at Charlotte, Charlotte, North Carolina, 28223-0001, USA; E-mail: tglucas@email.uncc.edu.

Throughout this paper, $R$ will denote an integral domain with quotient field $K$. For a pair of fractional ideals $I$ and $J$ of a domain $R$ we let $(J: I)$ denote the set $\{t \in K \mid t I \subseteq J\}$. Often, we shall use $I^{-1}$ in place of $(R: I)$. Recall that the " $v$ " of a fractional ideal $I$ is the set $I_{v}=(R:(R: I))$ and the " $t$ " of $I$ is the set $I_{t}=\bigcup J_{v}$ with the union taken over all finitely generated fractional ideals contained in $I$. An ideal $I$ is divisorial if $I=I_{v}$, and $I$ is a $t$-ideal if $I=I_{t}$.

Let $R$ be an integral domain and let $M$ be an $R$-module. Then the trace of $M$ is the ideal generated by the set $\{f m \mid f \in \operatorname{Hom}(M, R)$ and 
$1 m \in M\}$. For a fractional ideal $I$ of $R$, the trace is simply the product of

$2 \quad I$ and $I^{-1}$. We call an ideal of $R$ a trace ideal of $R$ if it is the trace of some

$3 R$-module. An elementary result due to Bass is that if $J$ is a trace ideal of $4 R$, then $J J^{-1}=J$; i.e., $J^{-1}=(J: J)$ (Bass, 1963, Proposition 7.2). It follows 5 that $J$ is a trace ideal if and only if $J^{-1}=(J: J)$. (Such ideals are also 6 referred to as being "strong"; see, for example, Barucci, 1986.) In 1987, 7 Anderson, Huckaba and Papick proved that if $I$ is a noninvertible ideal 8 of a valuation domain $V$, then $I(V: I)$ is prime (Anderson et al., 1987, 9 Theorem 2.8). Later in the same year, Fontana, Huckaba and Papick 10 began the study of the "trace property" and "TP domains". A domain $11 R$ is said to satisfy the trace property (or to be a TP domain) if for each $12 R$-module $M$, the trace of $M$ is equal to either $R$ or a prime ideal of $R$ 13 (Fontana et al., 1987, page 169). Among other things, they showed that 14 each valuation domain satisfies the trace property (Fontana et al., 1987, 15 Proposition 2.1), and that if $R$ satisfies the trace property, then it has at 16 most one noninvertible maximal ideal (Fontana et al., 1987, Corollary 17 2.11). For Noetherian domains they proved that if $R$ is a Noetherian 18 domain, then it is a TP domain if and only if it is one-dimensional, has 19 at most one noninvertible maximal ideal $M$, and if such a maximal ideal 20 exists, then $M^{-1}$ equals the integral closure of $R$ (or, equivalently, $M^{-1}=(M: M)$ is a Dedekind domain) (Fontana et al., 1987, Theorem 3.5). In terms of pullbacks they proved that a Noetherian domain $R$ is a TP domain if and only if there is a Dedekind domain $T$, an ideal $I$ of $T$ and a subfield $F$ of $T / I$ such that $T / I$ is a finitely generated $F$-module and $R$ is the pullback in the following diagram

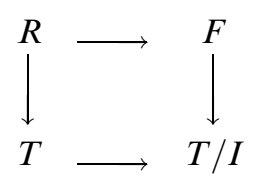

(Fontana et al., 1987, Theorem 3.6). In Sec. 2, Gabelli (1992) proved similar results about Mori domains. Specifically she showed that by replacing "integral closure" with "complete integral closure" and deleting the requirement that $T / I$ be finitely generated as a $F$-module, then the same lists of conditions (from Fontana et al., 1987, Theorems 3.5 and 3.6) characterize the class of Mori domains which satisfy the trace property (Gabelli, 1992, Theorem 2.9). Recall that a Mori domain is an integral domain which satisfies the ascending chain condition on divisorial ideals.

In 1988, Heinzer and Papick introduced the "radical trace property" declaring that an integral domain $R$ satisfies the radical trace property (or is an RTP domain) if for each noninvertible ideal $I, I I^{-1}$ is a radical ideal. 
For Noetherian domains, they proved that if $R$ is a Noetherian domain, then it satisfies the radical trace property if and only if $R_{P}$ is a TP domain for each prime ideal $P$ (Heinzer and Papick, Proposition 2.1). Gabelli extended this result to Mori domains (Gabelli, 1992, Theorem 2.14). She also gave a pullback characterization in the special case that the conductor between the domain in question and its complete integral closure is nonzero (Gabelli, 1992, Theorem 2.16).

According to Lucas (1996), a domain $R$ is said to satisfy the trace property for primary ideals (or to be a TPP domain), if for each primary ideal $Q$, either $Q$ is invertible or $Q Q^{-1}$ is prime. By Lucas (1996, Corollary 8), $R$ is a TPP domain if and only if for each primary ideal $Q$, either $Q Q^{-1}=\sqrt{Q}$, or $Q$ is invertible and $\sqrt{Q}$ is maximal. Also from Lucas (1996), $R$ is a PRIP domain if for each primary ideal $Q, Q^{-1}$ a ring implies $Q$ is prime. Note that in general, a primary ideal can be such that its inverse is a ring without the ideal being a trace ideal. In Kabbaj et al. (1999), the authors introduced the notion of an LTP domain as a domain with the property that for each trace ideal $I$ and each prime ideal $P$ minimal over $I, I R_{P}=P R_{P}$. In Kabbaj et al. (1999, Theorem 2), it was shown that a domain $R$ is an LTP domain if and only if each primary trace ideal is prime. In general, we have $\mathrm{RTP} \Rightarrow \mathrm{TPP} \Rightarrow \mathrm{LTP}$ and PRIP $\Rightarrow \mathrm{LTP}$ (Lucas, 1996, Theorem 4 and Kabbaj et al., 1999, Corollary 3). For Prüfer domains, all four are equivalent (Lucas, 1996, Theorem 23 and Kabbaj et al., 1999, Theorem 10); and for Mori domains, $\mathrm{PRIP} \Rightarrow \mathrm{RTP} \Leftrightarrow \mathrm{TPP} \Leftrightarrow \mathrm{LTP}$ (Kabbaj et al., 1999, Theorem 18), but there are examples of Mori RTP domains which do not satisfy PRIP (Lucas 1996, Example 30). In general, we have been unable to determine whether each TPP domain is an RTP domain, or whether each LTP domain is an TPP domain (or RTP domain).

The main concern of this paper is to consider diagrams of the form

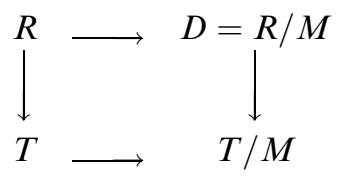

where $M$ is a prime ideal of $R$ with the quotient field of $D$ contained in $T / M$. While our ultimate goal is to completely characterize when one can say that $R$ is an "XTP" domain if and only if both $D$ and $T$ are "XTP" domains, with "XTP" being any one of TP, RTP, TPP or LTP, the current ones are far more modest. In the special case where $M$ is a maximal ideal of $T$, we show that $R$ is an RTP (TPP) [LTP] domain if and only if both $T$ and $D$ are RTP (TPP) [LTP] domains. In a somewhat less restricted situation, we show that if $M$ is a radical ideal 
of $T$ where each minimal prime of $M$ in $T$ is maximal, then $R$ is an LTP domain if and only if both $T$ and $D$ are LTP domains. By further requiring that the intersection of the minimal primes of $M$ be irredundant, we prove that a similar conclusion holds for both RTP and TPP. Note that it is known that if $R$ is an "XTP" domain and $P$ is a prime ideal of $R$, then $R / P$ is an "XTP" domain (Lucas 1996, Theorems 3 and 9 and Kabbaj et al., 1999, Theorem 4). While the restriction that each minimal prime of $M$ in $T$ be a maximal ideal of $T$ is not needed to prove that $D$ is an "XTP" domain when $R$ is, it is somewhat necessary to have such a restriction in order to have that $T$ is an "XTP" domain when $R$ is. For example, let $V=F(x)+y F(x)[[y]], \quad T=F\left[x^{2}, x^{5}\right]+y F(x)[[y]] \quad$ and $R=F+y F(x)[[y]]$. Both $V$ and $R$ are TP domains (Fontana et al., 1987, Proposition 2.1 and Heinzer and Papick, Example 2.12). However, the ideal $Q=\left(x^{4}, x^{5}\right) T$ is a primary ideal of $T$ which is also a trace ideal but not prime (specifically, $(Q: Q)=(T: Q)=V)$. Thus $T$ is not even an LTP domain.

A field is trivially an RTP domain. While most of the results in this paper are true for fields, the emphasis is on integral domains that are not fields. To avoid having to add the phrase "but not a field" when it would be required, we will simply assume that $R$ is an integral domain which is not a field. We shall also assume that all of the ideals in question are nonzero.

Notation is standard as in Gilmer (1972). In particular, " $\subseteq$ " denotes containment and " $\subset$ " denotes proper containment.

\section{PRELIMINARY RESULTS}

We shall make use of a number of results concerning consequences of $I^{-1}$ being a ring and several other results more specific to dealing with trace properties. We collect a few of these results in this section. Many, but not all, of these results have appeared elsewhere.

Theorem 1. Let $R$ be an integral domain and let $I$ be an ideal of $R$ such that $I^{-1}$ is a ring. Then

(a) $I^{-1}=I_{v}^{-1}=\left(I_{v}: I_{v}\right)=\left(I I^{-1}: I I^{-1}\right)=\left(I I^{-1}\right)^{-1} \quad$ (Huckaba and Papick, 1982, Proposition 2.2).

(b) $\sqrt{I}^{-1}$ is a ring (Houston et al., 2000, Proposition 2.1). Moreover, $\sqrt{I}^{-1}=(\sqrt{I}: \sqrt{I})$ (Anderson, 1983, Proposition 3.3).

(c) $P^{-1}$ is a ring for each prime P minimal over I (Houston et al., 2000, Proposition 2.1 and Lucas, 1996, Lemma 13). Moreover, $P^{-1}=(P: P)($ Houston et al., 2000, Proposition 2.3). 
The next result is a variation on a result which appears in Fossum's book (Fossum 1973, Lemma 3.7). (See also, Lucas, 1996, Lemmas 0 and 1.)

Lemma 2. Let $R$ be an integral domain and let $Q$ be a primary ideal of $R$ with radical $P$. If $P$ does not contain $Q Q^{-1}$, then $\left(R: Q Q^{-1}\right)=\left(Q Q^{-1}\right.$ : $\left.Q Q^{-1}\right)=(Q: Q)$ and so $(R: I)=(Q: Q)$ for each ideal $I$ such that $Q \subset I \subseteq Q Q^{-1}$ and $I \notin P$.

There are (at least) two ways to characterize LTP domains in terms of primary ideals.

Theorem 3 (Kabbaj et al., 1999, Theorem 2). The following are equivalent for a domain $R$.

(1) $R$ is an LTP domain.

(2) For each noninvertible primary ideal $Q, Q(R: Q) R_{P}=P R_{P}$ where $P=\sqrt{Q}$

(3) If a primary ideal is also a trace ideal, then it is prime.

Recall that an integral domain is a TPP domain if and only if for each noninvertible primary ideal $Q, Q(R: Q)=P$ where $P$ is the radical of $Q$ (Lucas, 1996, Corollary 16). Obviously, each TPP domain satisfies the condition in statement (2) of Theorem 3. Thus each TPP domain is an LTP domain. Also, note that each PRIP domain satisfies statement (3) of Theorem 3. While we have not been able to show that there are LTP domains which are not TPP domains, we can show that there are LTP domains which are not PRIP domains. For example, consider the ring $R=F\left[\left[x^{3}, x^{4}, x^{5}\right]\right]$ where $F$ is a field (this is the ring of Example 30 in Lucas, 1996). The ideal $Q=\left(x^{3}, x^{4}\right)$ is primary but not prime and $Q^{-1}=F[[x]]$ is a ring. Thus $R$ is not a PRIP domain. However, note that $Q Q^{-1}=\left(x^{3}, x^{4}, x^{5}\right)$ is the maximal ideal of $R$ and $\left(Q Q^{-1}\right)^{-1}=F[[x]]$. By Fontana et al. (1987, Theorem 3.5), $R$ is an RTP domain. As every RTP domain is a TPP domain (Lucas, 1996, Theorem 4), $R$ is an LTP domain. (In fact, all three of RTP, TPP and LTP are equivalent for Noetherian domains (Lucas, 1996, Theorem 12 and Kabbaj et al., 1999, Theorem 18)).

Theorem 4. Let $R$ be an integral domain. If $R$ is an RTP domain, a TPP domain or a PRIP domain, then $R$ is an LTP domain (Lucas 1996, Theorem 4 and Kabbaj et al., 1999, Corollary 3). 
1090

Kabbaj, Lucas, and Mimouni

The next two results collect useful information concerning the prime ideals of an RTP, TPP and LTP domains.

Theorem 5. Let $P$ be a prime ideal of an integral domain $R$. If $R$ is an RTP (TPP) [LTP] domain, then both $R_{P}$ and $R / P$ are RTP (TPP) [LTP] domains (Lucas 1996, Theorems 3 and 9, and Kabbaj et al., 1999, Theorem 4, respectively).

Theorem 6 (Kabbaj et al., 1999, Theorem 5). Let R be an LTP domain. Then

(a) Each maximal ideal is a t-ideal.

(b) Each nonmaximal prime ideal is a divisorial trace ideal.

(c) Each maximal ideal is either idempotent or divisorial.

By Theorem 4, all three of the above statements in Theorem 6 also hold for the prime ideals of RTP, TPP and PRIP domains. For "new" results, we begin with the following lemma.

Lemma 7. Let $R \subset T$ be a pair of domains for which $B=(R: T)$ is not zero.

(1) If $J$ is a trace ideal of $T$ and $J B=J \cap B$, then $J B$ is a trace ideal of $R$.

(2) If $Q^{\prime}$ is an invertible primary ideal of $T$ whose radical in $T$ is maximal and incomparable with $B$, then $Q=Q^{\prime} \cap R$ is an invertible primary ideal of $R$ whose radical is a maximal ideal of $R$.

Proof. Since $B$ is an ideal of both $R$ and $T$, if $t \in(R: B)$, then $t B=t B T \subseteq R$. It follows that $(R: B)=(B: B)$.

Let $J$ be a trace ideal of $T$ for which $J B=J \cap B$. Then for each $u \in(R: J B)$, we have $u B \subseteq(T: J)=(J: J)$ and $u J \subseteq(R: B)=(B: B)$. Thus $u J B \subseteq J \cap B=J B$ and therefore, $J B$ is a trace ideal of $R$.

Let $Q^{\prime}$ be an invertible primary ideal of $T$ whose radical in $T$ is maximal and incomparable with $B$ and let $Q=Q^{\prime} \cap R$. Let $N^{\prime}$ denote the radical of $Q^{\prime}$ in $T$ and let $N=N^{\prime} \cap R$. That $Q$ is $N$-primary and $N$ is a maximal ideal of $R$ follows from Fontana (1973, Theorem 1.4 and Corollary 1.5). We also have $R_{N}=T_{N^{\prime}}$ and $Q R_{N}=Q^{\prime} T_{N^{\prime}}$. Since $N$ is a maximal ideal of $R$, it suffices to show that $\left(Q Q^{-1}\right) R_{N}=R_{N}$. As $B$ is an ideal of both $R$ and $T$, we have $Q B\left(T: Q^{\prime}\right) \subseteq B Q^{\prime}\left(T: Q^{\prime}\right)=B \subset R$. Hence $B\left(T: Q^{\prime}\right) \subseteq Q^{-1}$. Since $B$ and $N^{\prime}$ are incomparable ideals of $T, B T_{N^{\prime}}=T_{N^{\prime}}=R_{N}$. Thus 
$R_{N}=Q^{\prime}\left(T: Q^{\prime}\right) T_{N^{\prime}}=Q B\left(T: Q^{\prime}\right) R_{N} \subseteq\left(Q Q^{-1}\right) R_{N}=R_{N}$. Therefore, $Q$ is an invertible ideal of $R$.

Several authors have established the invertibility statement in 7(b) in more restrictive settings. See, for example, Costa et al. (1978) and Fontana and Gabelli (1996).

A little more can be said in the special case that $T=(I: I)$ for some ideal $I$ of $R$. In particular, we have the following.

Lemma 8 (Kabbaj et al., 1999, Lemma 6). Let I be a trace ideal of an integral domain $R$ and let $J$ be an ideal of $(I: I)$.

(a) If $J$ contains $I$, then $J \cap R$ is a trace ideal of $R$.

(b) If $J$ is a trace ideal of $(I: I)$, then $I J$ is a trace ideal of $R$.

The last of our preliminary results deals with certain invertible ideals in an LTP domain.

Lemma 9. Let $R$ be an LTP domain and let I be a radical ideal of $R$ for which each minimal prime is a maximal ideal. If I is invertible, then each prime that contains $I$ is invertible and is each ideal whose radical contains I and the intersection $\bigcap\left\{M_{\alpha} \in \operatorname{Max}(R) \mid I \subseteq M_{\alpha}\right\}$ is irredundant.

Proof. Assume $I$ is invertible and let $N$ be a prime containing $I$. Then $N$ is a maximal ideal of $R$ and $I R_{N}=N R_{N}$. It follows that $N R_{N}$ is invertible. Thus $N \neq N^{2}$ and hence it is divisorial by Theorem 6 . We also have $(N: N)=R$ as $(N: N) \subseteq\left(N R_{N}: N R_{N}\right)=R_{N}$ and $(N: N) \subseteq(R: N) \subseteq R_{M}$ for each maximal ideal $M \neq N$. As $N$ is divisorial we have $(R: N) \neq R=(N: N)$. It follows that $N$ must be an invertible ideal.

Let $M_{\beta}$ be a minimal prime of $I$. As $I$ is a radical ideal and $M_{\beta}$ is minimal over $I, I R_{M_{\beta}}=M_{\beta} R_{M_{\beta}}$. Since $M_{\beta}$ is invertible, there is an element $s \in R \backslash M_{\beta}$ such that $s M_{\beta} \subseteq I$. It follows that $s$ is contained in each maximal ideal $M_{\alpha}$ that contains $I$ except $M_{\beta}$. Thus the intersection $\bigcap\left\{M_{\alpha} \in\right.$ $\left.\operatorname{Max}(R) \mid I \subseteq M_{\alpha}\right\}$ is irredundant.

Let $B$ be an ideal of $R$ with $\sqrt{B}=I$. As each minimal prime of $B$ is also a minimal prime of $I$, each minimal prime of $B$ is an invertible maximal ideal of $R$. By Theorem 1, each prime minimal over a trace ideal is also a trace ideal so no maximal ideal of $R$ can contain $B B^{-1}$. Hence, $B$ is invertible.

Houston et al. (2000, Example 5.1) shows that if a domain $R$ is not an LTP domain, then it may contain an invertible radical ideal all of whose minimal primes are maximal ideals with inverse equal to $R$. 


\section{M MAXIMAL IN $T$}

Let $T$ be a domain with a maximal ideal $M$ and let $D$ be a domain contained in $T / M$. Let $R$ be the pullback of the following diagram:

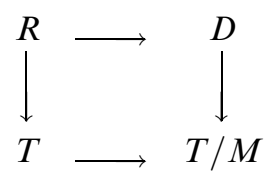

We begin with a lemma concerning the primary ideals of $R$.

Lemma 10. For diagram $\square_{1}$, 
(a) If $Q$ is a primary ideal of $R$ which is neither contained in $M$ nor comaximal with $M$, then $Q$ contains $M$.

(b) If $B$ is an ideal of $R$ that contains an $M$-primary ideal and is not contained in $M$, then $B T=T$ and $B$ contains $M$.

Proof. Let $Q$ be a primary ideal of $R$ which is neither contained in $M$ nor comaximal with $M$ and let $P=\sqrt{Q}$. Since $M$ is a maximal ideal of $T$, a prime ideal of $R$ is either comparable to $M$ or comaximal with $M$ in $R$ (Fontana, 1973, Theorem 1.4). Since $Q+M \neq R$, we must have $P$ and $M$ comparable. As $M$ does not contain $Q$, we have $M \subset P$. Thus $M=M T \subset P T$. Again since $M$ is a maximal ideal of $T$, we have $P T=T$. As $Q$ is $P$-primary, we also have $Q T=T$. It follows that $M=M T=$ $M Q T=M Q \subset Q$.

Let $B$ be an ideal of $R$ that contains an $M$-primary ideal $Q$. Since $M$ is a maximal ideal of $T$, it is the only maximal ideal of $T$ that contain $Q$. Hence, if $M$ does not contain $B$, then no maximal ideal of $T$ can contain $B$. It follows that $B T=T$ and that $M \subset B$.

Theorem 11. For diagram $\square$, R is an LTP domain if and only if both $T$ and $D$ are LTP domains.

Proof. $\quad(\Rightarrow)$ Assume $R$ is an LTP domain. By Theorem 3 (i.e., Kabbaj et al., 1999, Theorem 2), it suffices to show that only prime ideals can be both primary ideals and trace ideals of $T$. To this end, let $Q^{\prime}$ be a primary ideal of $T$ which is also a trace ideal. Let $I=Q^{\prime} \cap M, P^{\prime}=\sqrt{Q^{\prime}}$ and $P=P^{\prime} \cap R$.

If $Q^{\prime}+M=T$, then $I=Q^{\prime} M$. Hence it follows from Lemma 7 that $I$ is a trace ideal of $R$. We also have $P+M=R$ with $P$ a minimal prime of $I$. Since $R$ is an LTP domain and $M$ does not contain $P$, we have $P^{\prime} T_{P^{\prime}}=P R_{P}=I R_{P}=M Q^{\prime} R_{P} \subseteq Q^{\prime} T_{P^{\prime}} \subseteq P^{\prime} T_{P^{\prime}}$. Since $Q^{\prime}$ is $P^{\prime}$-primary, we have $Q^{\prime}=P^{\prime}$.

If $Q^{\prime}+M \neq T$, then $Q^{\prime} \subseteq M, I=Q^{\prime}$ and $P=P^{\prime}$. Thus $Q^{\prime}$ is a primary trace ideal of $R$. As $R$ is an LTP domain, $Q^{\prime}=P^{\prime}$.

$(\Leftarrow)$ Assume both $T$ and $D$ are LTP domains and let $I$ be a trace ideal of $R$. By Theorem 3, we may assume that $I=Q$ is primary with radical $P$.

Case 1. $Q \subseteq M$.

If $P \neq M$, then $Q$ is also a primary ideal of $T$. As $P$ is not a maximal ideal of $T, Q(T: Q) \subseteq P$ by Theorem 3. It follows that $Q$ is also a trace ideal of $T$. Hence $Q=P$.

If $P=M$, then $Q T$ is an $M$-primary ideal of $T$. Hence we either have $Q(T: Q)=M$ or $Q(T: Q)=T$. If the former, $(Q: Q)=(R: Q)=(T: Q)$ and 
1 therefore, $Q=M=P$. If the latter, $M=M Q(T: Q) \subseteq Q(R: Q)=Q$ so 2 again we have $Q=M=P$.

Case 2. $Q+M=R$.

Let $J=Q(T: Q)$. Then $J$ is a trace ideal of $T$ and $J+M=T$. Hence $J \cap M=J M$ and there is a unique prime $P^{\prime}$ of $T$ that contracts to $P$. As $Q$ is trace ideal of $R$, it contains $J M$. Since $M$ and $P^{\prime}$ are comaximal ideals of $T, P^{\prime}$ must be minimal over $J$. Hence $J M R_{P}=J M T_{P^{\prime}} \subseteq Q R_{P} \subseteq P R_{P}=$ $P^{\prime} T_{P^{\prime}}=J T_{P^{\prime}}=J M T_{P^{\prime}}$. It follows that $Q R_{P}=P R_{P}$ and therefore, $Q=P$.

Case 3. $Q \nsubseteq \nsubseteq M$ and $Q+M \neq R$.

By Lemma 10, we must have $M \subset Q$. Thus by Houston et al. (2000, Proposition 6), we have that $(D: \bar{Q})=\overline{(R: Q)}=\overline{(Q: Q)}=(\bar{Q}: \bar{Q}$. Since $D$ is an LTP domain, $\bar{Q}=\bar{P}$. It follows that $Q=P$.

Theorem 12. For diagram $\square_{1}, R$ is a TPP domain if and only if both $T$ and $D$ are TPP domains.

Proof. Assume $R$ is a TPP domain and let $Q^{\prime}$ be a $P^{\prime}$-primary ideal of T. Let $Q=Q^{\prime} \cap R$ and $P=P^{\prime} \cap R$. Thus $M Q^{\prime} \subseteq Q$ and $M P^{\prime} \subseteq P$. As $M Q^{\prime}\left(T: Q^{\prime}\right) \subseteq M, M\left(T: Q^{\prime}\right) \subseteq(R: Q)$ and therefore, $M^{2} Q^{\prime}\left(T: Q^{\prime}\right) \subseteq$ $Q(R: Q) \cap M$.

Case 1. $Q^{\prime}+M=T$.

In this case $Q+M=R$ and for each maximal ideal $N^{\prime}$ containing $Q^{\prime}$, $Q R_{N}=Q T_{N^{\prime}}=Q^{\prime} T_{N^{\prime}}$ and $P R_{N}=P T_{N^{\prime}}=P^{\prime} T_{N^{\prime}}$ where $N=N^{\prime} \cap R$. It follows that $(R: Q) \subseteq\left(T: Q^{\prime}\right)$. If $Q$ is invertible, so is $Q^{\prime}$. On the other hand, if $Q(R: Q)=P$, then $P^{\prime} T_{N^{\prime}}=P T_{N^{\prime}}=Q(R: Q) T_{N^{\prime}}=Q^{\prime}\left(T: Q^{\prime}\right) T_{N^{\prime}}$ and it follows that $Q^{\prime}\left(T: Q^{\prime}\right)=P^{\prime}$.

Case 2. $Q^{\prime} \subseteq M$.

In this case we have $Q=Q^{\prime}$. Hence $Q(R: Q) \subseteq Q^{\prime}\left(T: Q^{\prime}\right)$. If $Q(R: Q)=R$, then $Q^{\prime}\left(T: Q^{\prime}\right)=T$. On the other hand, if $Q(R: Q)=P$, then we at least have $P^{\prime}=P \subseteq Q^{\prime}\left(T: Q^{\prime}\right)$. If $P \neq M$, the fact that $M^{2} Q^{\prime}\left(T: Q^{\prime}\right)$ is contained in $Q(R: Q)$, implies $Q^{\prime}\left(T: Q^{\prime}\right) \subseteq P^{\prime}=P$. If $P=M$, we have that either $Q^{\prime}\left(T: Q^{\prime}\right)=T$ or $Q^{\prime}\left(T: Q^{\prime}\right)=M$.

$(\Leftarrow)$ Assume both $T$ and $D$ are TPP domains and let $Q$ be a $P$ primary ideal of $R$.

\section{Case 1. $P \subset M$.}

In this case $P$ is also a prime ideal of $T$ and $Q$ is a $P$-primary ideal of $T$. Since $T$ is a TPP domain, $Q(T: Q)=P$. Hence we have $Q(R: Q)=P$. 
Case 2. $P+M=R$.

Since $P+M=R$, there is a unique prime ideal $P^{\prime}$ of $T$ that contracts to $P$ and a unique $P^{\prime}$-primary ideal $Q^{\prime}$ that contracts to $Q$. Since $M$ is a common ideal of $R$ and $T, M Q^{\prime} \subseteq M \cap Q^{\prime} \subset Q$ and $M Q^{\prime}\left(T: Q^{\prime}\right) \subseteq M$. contains $M^{2}$ so we also have that $Q$ is invertible. If $Q^{\prime}$ is not invertible, then $Q^{\prime}\left(T: Q^{\prime}\right)=P^{\prime}$ since $T$ is a TPP domain. Thus $M^{2} P^{\prime} \subseteq Q(R: Q)$. For each maximal ideal $N^{\prime}$ containing $Q^{\prime}$, we have $Q R_{N}=Q T_{N^{\prime}}=Q^{\prime} T_{N^{\prime}}$ where $N=N^{\prime} \cap R$. It follows that $(R: Q) \subseteq\left(T: Q^{\prime}\right)$. Hence, $Q(R: Q) \subseteq Q^{\prime}$ $\left(T: Q^{\prime}\right) \cap R=P^{\prime} \cap R=P$. By localizing at the maximal ideals that contain $Q$ we see that $Q=P$.

Case 3. $M \subset P$.

By Lemma 10 we also have $M \subset Q$. Hence $(D: \bar{Q}) \bar{Q}=\overline{(R: Q)} \bar{Q}$. Since $D$ is a TPP domain we either have that $\bar{Q}$ is invertible or that $\bar{Q}(D: \bar{Q})=\bar{P}$. If the former, $Q$ is invertible, and if the latter, $Q(R: Q)=P$.

Case 4. $P=M$.

If $Q(T: Q)=M$, we are done. So we may assume that $Q(T: Q)=T$. In this case we will have $M=M Q(T: Q) \subseteq Q(R: Q)$. If $D$ is a field, this is all we need. Thus we may further assume that $D$ is not a field. By way of contradiction, assume $Q(R: Q)$ properly contains $M$. If $Q(R: Q)=R$, then each ideal that properly contains $M$ has inverse equal to $R$. But if $B$ is an ideal of $R$ which properly contains $M$, then $(D: \bar{B})=\overline{(R: B)}$ (Houston et al., 2000, Proposition 6). Since we have assumed that $D$ is a TPP domain which is not a field, it follows that we cannot have $Q(R: Q)=R$. Let $t \in Q(R: Q) \backslash M$ and set $I=t^{2} R+Q$ and $B=I(R: I)$. By Lemma $10, B$ contains $M$ and is a trace ideal of $R$. Thus $(D: \bar{B})=\overline{(R: B)})=\overline{(B: B)}=(\bar{B} ; \bar{B})$. Since a TPP domain is also an LTP domain, $\bar{B} D_{N}=\bar{N} D_{N}$ for each prime $N$ minimal over $B$. Hence $B R_{N}=N R_{N}$. Thus we have elements $a \in(R: B)=(B: B), q \in Q$ and $s \in R \backslash N$, such that $s t=a t^{2}+q$ with $a t \in N$. Hence $q=t(s-a t)$. This is impossible since $Q$ is $M$-primary and neither $t$ nor $s-a t$ is in $M$.

Theorem 13. For diagram $\square_{1}, R$ is an RTP domain if and only if both $T$ and $D$ are RTP domains.

Proof. $\quad(\Rightarrow)$ Assume $R$ is an RTP domain and let $J$ be a trace ideal of $T$. Let $I=J \cap M$. If $J$ and $M$ are comaximal, then $I=J M$. If $J$ and $M$ are not comaximal, then $J \cap M=J$. In either event, $I$ is a trace ideal of $R$.

Case 1. $J+M=T$. 
In this case for each maximal ideal $N^{\prime}$ containing $J, I R_{N}=I T_{N^{\prime}}=$ $J T_{N^{\prime}}$ where $N=N^{\prime} \cap R$. As $I$ is a radical ideal of $R, J$ is a radical ideal of $T$.

Case 2. $J \subseteq M$.

In this case $I=J$ is a radical ideal of $R$. Since $M$ contains $J$ it contains the radical of $J$ in $T$. Thus $J$ is a radical ideal of both $T$ and $R$.

$(\Leftarrow)$ Assume both $T$ and $D$ are RTP domains and let $I$ be a trace ideal of $R$. Let $J=I(T: I)$. Then $J$ is a trace ideal of $T$ and as such it is a radical ideal of $T$.

Case 1. $J \subseteq M$.

In this case $I=J$ is a radical ideal of $T$. So it is also a radical ideal of $R$.

Case 2. $I+M=R$.

In this case we obviously also have $J+M=T$. Hence $J \cap M=J M=\subseteq I$. As no maximal ideal of $R$ can contain both $I$ and $M, J R_{N}=J M R_{N} \subseteq I R_{N} \subseteq J R_{N}$ for each maximal ideal $N$ (of $R$ ) that contains $I$. As $J$ is a radical ideal of $T, I$ is a radical ideal of $R$. Moreover, we must have $J \cap R=I$.

Case 3. $I \subseteq M, I+M \neq R$ but $J+M=T$.

If $J=T$, then we have $M=M J \subseteq I$. If $I=M$, there is nothing to prove. If $I$ properly contains $M$, then we have $;(D: \bar{I})=\overline{(R: I)})=$ $\overline{(I: I)})=(\bar{I}: \bar{I})$. Since $D$ is an RTP domain, $\bar{I}$ is a radical ideal of $D$ and it follows that $I$ is a radical ideal of $R$.

If $I$ does not contain $M$, then $J \neq T$. Set $A=J \cap R$. Then we have $A+M=R$ so that $A \cap M=A M \subseteq I$. Set $B=I+M$. Then $B$ is trace ideal of $R$ that does contain $M$. So $B$ is a radical ideal of $R$. Since $A+M=R$, we also have $A+B=R$. Hence $A B=A \cap B$ is a radical ideal of $R$ that both contains and is contained in $I$. Thus $I=A \cap B$ is a radical ideal of $R$.

Case 4. $I \subseteq M$ but $J \not \subset M$.

In this case we have $J+M=T$. Hence $J \cap M=J M \subseteq I$. As both $J$ and $M$ contain $I$, we have $I=J \cap M$. Since both $J$ and $M$ are radical ideals of $T, I$ is a radical ideal of $R$.

If $R$ is an RTP Prüfer domain, then for each ideal $I$, the ring $(I: I)$ is an RTP Prüfer domain (Lucas, 1996, Corollary 24). Moreover, for a prime ideal $P, P$ is a maximal ideal of $(P: P)$. Also, if $R$ is an RTP Mori domain and $I$ is a trace ideal of $R$, then $(I: I)$ is an RTP domain (Kabbaj et al., 1999, Corollary 19). On the other hand, Kabbaj et al. (1999,

42 Example 15) gives an example of an RTP domain with an ideal $I$ such 
that $(I: I)$ is not an RTP domain . The ideal in that example is not a trace ideal of $R$. It remains an open question as to whether $(I: I)$ has the same trace property as $R$ when $I$ is a trace ideal of $R$. By Theorems 11, 12 and 13 we can make the following statement.

Corollary 14. Let $P$ be a prime ideal of a domain $R$. If $P$ is a maximal ideal of $(P: P)$, then $R$ is an LTP $(T P P)[R T P]$ domain if and only if both $(P: P)$ and $R / P$ are $L T P(T P P)[R T P]$ domains.

For the TP property, we need to make some further assumption(s) in order to get results which correspond to those we have established for RTP, TPP and LTP. In our next result, we shall add the restriction that $T$ is quasilocal. Later we shall establish a similar result under the assumption that $T$ is a Dedekind domain. Note that in this later result, we shall not require that $M$ be a maximal ideal of $T$, but only that the quotient field of $D$ be contained in $T / M$. Also, we shall give an example of a pullback $R$ where $R$ is not a TP domain even though $M$ is a maximal ideal of $T$ and both $T$ and $D$ are TP domains (Example 33).

Recall from Cahen and Lucast (1997, Corollary 11), that a domain is a TP domain if and only if it is an RTP domain for which the noninvertible primes are linearly ordered.

Theorem 15. For diagram $\square_{1}$, further assume that $T$ is quasilocal. Then $R$ is a TP domain if and only if both $T$ and $D$ are TP domains.

Proof. $\quad(\Rightarrow)$ Assume $R$ is TP domain. That $D$ is a TP domain is a consequence of Cahen and Lucas (1997, Corollary 11). Let $J$ be a trace ideal of $T$. Since $T$ is quasilocal we have $J \subseteq M$, and hence $J$ is also a trace ideal of $R$. Hence $J$ is a prime ideal of $R$. As $M$ contains $J, J$ is a prime ideal of $T$ as well.

$(\Leftarrow)$ Assume that both $T$ and $D$ are TP domains. Since $T$ is quasilocal and $M$ is the maximal ideal of $T$, every ideal of $R$ compares with $M$. By Theorem 13, $R$ is an RTP domain. Thus by Cahen and Lucas (1997, Corollary 11) all we need to show is that the noninvertible primes of $R$ are linearly ordered. For a pair of prime ideals of $R$, each is comparable with $M$. Thus since $T$ is a TP domain, if either prime is contained in $M$, then the two are comparable. On the other hand if neither is contained in $M$, then both properly contain $M$ and their images in $D$ will be noninvertible (Fontana and Gabelli, 1996, Corollary 1.7) and therefore comparable since $D$ is a TP domain. It follows that $R$ is a TP domain. 
1098

Kabbaj, Lucas, and Mimouni

Recall from Hedstrom and Houston (1978) that a domain $R$ is pseudo-valuation domain if it is quasilocal and shares its maximal ideal with a valuation domain which necessarily must contain $R$ and be unique. In terms of pullbacks, $R$ is a pseudo-valuation domain if and only if there is a valuation domain $V$ with maximal ideal $M$ and a subfield $F$ of $V / M$ such that $R$ is the pullback in the following diagram

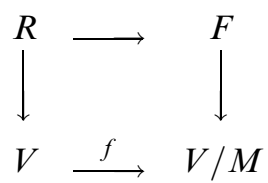

(Anderson and Dobbs, 1980, Proposition 2.6). It follows that each pseudo-valuation domain is a TP domain (see Heinzer and Papick, Example 2.12) for the classical " $D+M$ " case where $V=L+M$ and $R=F+M$.).

Corollary 16. Let $P$ be a prime ideal of a domain $R$. If $(P: P)$ is quasilocal with maximal ideal $P$, then $R$ is a TP domain if and only if both $(P: P)$ and $(P: P) / P$ are $T P$ domains.

\section{M A RADICAL IDEAL $T$}

Now consider the following situation. Let $T$ be a domain with a radical ideal $M$ for which $T / M$ contains a field $F$ and each minimal prime of $M$ is a maximal ideal of $T$. Let $S$ be the pullback of the following diagram:

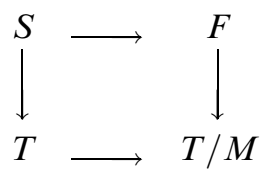

[Note that while we are primarily concerned with the case where $M$ is NOT a maximal ideal of $T$, we shall not make such an assumption in this section even though we have taken care of the case that $M$ is a maximal ideal of $T$ above in Theorems 11, 12 and 13.]

Theorem 17. For diagram $\square_{2}, S$ is an LTP domain if and only if $T$ is an $L T P$ domain. Moreover, if $S$ is an LTP domain and $T=(M: M)$, then for each maximal ideal $M_{\alpha}$ containing $M, M_{\alpha}$ is either idempotent or invertible as an ideal of $T$. 
1 Proof. We start by proving the second statement. So assume $S$ is an 2 LTP domain and that $T=(M: M)$ (with $M$ a radical ideal of $T$ where 3 each minimal prime is maximal). Let $M_{\alpha}$ be a maximal ideal of $T$ that 4 contains $M$. We may assume $M_{\alpha}$ is not an invertible ideal of $T$, which means it is a trace ideal of $T$. Thus by Kabbaj et al. (1999, Lemma 6) (Lemma 8 above), we have that $M M_{\alpha}$ is a trace ideal of $S$. But $M M_{\alpha}$ is an $M$-primary ideal of $S$, hence we have $M=M M_{\alpha}$ since $S$ is an LTP domain. By checking locally in $T$ we see that $M_{\alpha}$ is idempotent.

$(\Rightarrow)$ Assume $S$ is an LTP domain and let $Q^{\prime}$ be a primary ideal of $T$ which is also a trace ideal of $T$. Let $Q=Q^{\prime} \cap S, P^{\prime}=\sqrt{Q^{\prime}}$ and $P=P^{\prime} \cap S$. Since $M$ is an ideal of both $T$ and $S, Q^{\prime} M \subseteq Q$. We have three cases to consider.

Case 1. $P+M=S$.

In this case $Q^{\prime} M=Q^{\prime} \cap M=Q \cap M$ is a trace ideal of $S$. Since $S$ is an LTP domain and $P$ is minimal over $Q^{\prime} M$, we have $Q^{\prime} T_{P^{\prime}}=Q S_{P}=Q^{\prime}$ $M S_{P}=P S_{P}=P^{\prime} T_{P^{\prime}}$. Hence $Q^{\prime}=P^{\prime}$.

\section{Case 2. $P \subset M$.}

Since $M Q^{\prime} \subseteq Q, M(S: Q) \subseteq\left(T: Q^{\prime}\right)$. As $S$ is an LTP domain and $P$ is not a maximal prime, $Q(S: Q)=P$. It follows that $M P=M Q(S: Q) \subseteq Q^{\prime}$ $\left(T: Q^{\prime}\right)=Q^{\prime}$. Since $P \neq M, M P S_{P}=P S_{P}=P^{\prime} T_{P^{\prime}}$. Thus $Q^{\prime} T_{P^{\prime}}=P^{\prime} T_{P^{\prime}}$ and it follows that $Q^{\prime}=P^{\prime}$.

Case 3. $P=M$.

In this case the ideal $M Q^{\prime}$ is an $M$-primary ideal of $S$. Since $S$ is an LTP domain, we have $M \subseteq M Q^{\prime}\left(S: M Q^{\prime}\right)$. Hence $M \subseteq Q^{\prime}\left(M\left(T: M Q^{\prime}\right)\right) \subseteq$ $Q^{\prime}\left(T: Q^{\prime}\right)=Q^{\prime}$. But $M T_{P^{\prime}}=P^{\prime} T_{P^{\prime}}$ since $M$ is a radical ideal of $T$ and $P^{\prime}$ is minimal over $M$. Therefore we again have $Q^{\prime}=P^{\prime}$.

$(\Leftarrow)$ Assume $T$ is an LTP domain and let $Q$ be a primary ideal of $S$ which is also a trace ideal. Let $P=\sqrt{Q}$ and $J=Q(T: Q)$. Then $J M \subseteq Q(S: Q)=Q$.

Case 1. $P \neq M$.

Since $P \neq M$, there is a unique prime $P^{\prime}$ of $T$ that contracts to $P$ and $P^{\prime}$ must be minimal over $J$. As $J$ is a trace ideal of $T$ and $P^{\prime}$ does not contain $M, J M T_{P^{\prime}}=J T_{P^{\prime}}=P^{\prime} T_{P^{\prime}}$. Furthermore, $Q S_{P}=Q T_{P^{\prime}}$ and $P S_{P}=P^{\prime} T_{P^{\prime}}$. Hence $Q S_{P}=P S_{P}$ and it follows that $Q=P$.

Case 2. $P=M$.

Let $M_{\alpha}$ be a maximal ideal of $T$ that contains $M$. Since each minimal prime of $M$ is a maximal ideal of $T, M_{\alpha}$ is minimal over $M$ and therefore, $M T_{M_{\alpha}}=M_{\alpha} T_{M_{\alpha}}$. Since $Q$ is a trace ideal of $S$ and is contained 42 in $M,(S: Q)=(Q: Q)$ contains $T$. Thus $Q$ is an ideal of $T$. As $Q$ is 
$1 \quad M$-primary (as an ideal of $S$ ), it suffices to show that $Q T_{M_{\alpha}}=M T_{M_{\alpha}}$. Let $2 Q^{\prime}=Q T_{M_{\alpha}} \cap T$. By way of contradiction assume $Q^{\prime} \neq M_{\alpha}$. Since $M_{\alpha}$ is a 3 maximal ideal of $T$ and $Q^{\prime}$ is $M_{\alpha}$-primary, $\left(T: Q^{\prime}\right) T_{N^{\prime}}=T_{N^{\prime}}$ for each 4 maximal ideal $N^{\prime} \neq M_{\alpha}$. Thus $M_{\alpha} Q\left(T: Q^{\prime}\right) T_{N^{\prime}}=Q\left(T: Q^{\prime}\right) T_{N^{\prime}}=Q T_{N^{\prime}} \subseteq$ $5 M T_{N^{\prime}}$. If $Q^{\prime}$ is an invertible ideal of $T$, then $M_{\alpha} Q^{\prime}\left(T: Q^{\prime}\right)=M_{\alpha}$ and

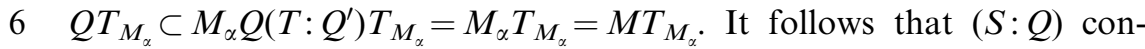
7 tains $M_{\alpha}\left(T: Q^{\prime}\right)$ and we get a contradiction since $Q(S: Q)=Q$ and $8 Q M_{\alpha}\left(T: Q^{\prime}\right) T_{M_{\alpha}}$ properly contains $Q T_{M_{\alpha}}$. If $Q^{\prime}$ is not invertible, then $9 Q^{\prime}\left(T: Q^{\prime}\right)=M_{\alpha}$ and it follows that $Q T_{M_{\alpha}} \subset Q\left(T: Q^{\prime}\right) T_{M_{\alpha}}=M_{\alpha} T_{M_{\alpha}}$. In 10 this case $(S: Q)$ contains $\left(T: Q^{\prime}\right)$ and we get a contradiction since

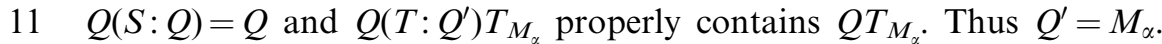
12 Since $M_{\alpha}$ was an arbitrary maximal ideal of $T$ that contains $M$ and $13 Q$, we have $Q=M$.

For diagram $\square_{2}$, let $D$ be a domain with quotient field $F$ and let $R$ be the pullback of the following diagram:

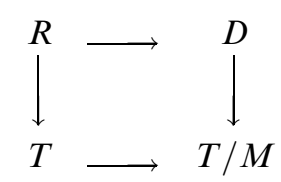

By combining Theorems 11 and 17 we have the following corollary.

Corollary 18. In diagram $\square_{3}, R$ is an LTP domain if and only if both $T$ and $D$ are LTP domains.

In general, we have not been able to extend the equivalence in Theorem 17 to either TPP domains or RTP domains. However, we have been successful if we also require that $M$ is an irredundant intersection of its minimal primes (and each such minimal prime is a maximal ideal of $T)$. This is the subject of our next section.

\section{M AN IRREDUNDANT INTERSECTION}

Let $T$ be a domain with a radical ideal $M$ which is an irredundant intersection of its minimal primes and for which each such minimal prime is a maximal ideal of $T$. Let $F$ be a field contained in $T / M$ and let $S$ be the pullback of the following diagram 


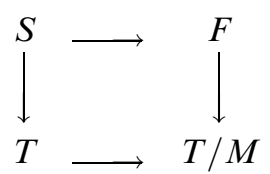

[As in the previous section we will not assume that $M$ cannot be a maximal ideal of $T$. On the contrary, that is simply a very special case that matches our assumption for this section.]

Recall from above that $\mathscr{U}$ denotes the set of prime ideals of $T$ which are minimal over $M$. For each ideal $J$ of $T$, we let $J_{d}=\bigcap\left\{M_{\alpha} \in \mathscr{M} \mid J \nsubseteq M_{\alpha}\right\}$ (=T if no such $M_{\alpha}$ s exist).

Lemma 19. Let $T$ and $S$ be the rings in diagram $\square_{4}$ and let $J$ be an ideal of $T$ and $I=J \cap J_{d}$. Then

(a) $I=J J_{d}$ is an ideal of $S$ and for each maximal ideal $N^{\prime}$ containing $J, I T_{N^{\prime}}=J T_{N^{\prime}}$. Moreover, if $N=N^{\prime} \cap S$ is not equal to $M$, then $I S_{N}=I T_{N^{\prime}}=J T_{N^{\prime}}$

(b) $J$ is a radical ideal of $T$ if and only if $I$ is a radical ideal of $S$.

(c) $J_{d} J(T: J) M=M I(T: J) \subseteq I(R: I)$.

(d) If $J$ is a trace ideal of $T$, then $I$ is a trace ideal of $S$.

(e) If $J=Q^{\prime}$ is a $P^{\prime}$-primary ideal of $T$, then $J J_{d}=Q^{\prime} J_{d} \subseteq Q=Q^{\prime} \cap S$ with equality if $J+M \neq T$.

Proof. Since the set of maximal ideals of $T$ that contain $M$ is irredundant, $J$ and $J_{d}$ are comaximal. Hence $I=J \cap J_{d}=J J_{d}$. It follows that if $N^{\prime}$ is a maximal ideal of $T$ that contains $J$, then $I T_{N^{\prime}}=J T_{N^{\prime}}$. If $N=N^{\prime} \cap S$ is not $M$, then $S_{N}=T_{N^{\prime}}$ and we also have $I S_{N}=I T_{N^{\prime}}=J T_{N^{\prime}}$.

Obviously, if $J$ is a radical ideal of $T$, then $I$ is a radical ideal of $S$. For the converse, note that if $P^{\prime}$ is a prime ideal of $T$ that contains $I$ and does not contain $M$, then $I \subseteq P^{\prime} \cap M=P \cap M$ where $P=P^{\prime} \cap R$. It follows that $I$ is also a radical ideal of $T$. Thus by (a), $J$ is a radical ideal of $T$.

Since $M$ is an ideal of $T, M J(T: J) \subseteq M$. From this it is easy to see that $M I(T: J) \subseteq I(R: I)$.

Assume $J$ is a trace ideal of $T$. Let $J_{1}$ denote the intersection of those maximal ideals which are not invertible and contain $M$ and not $J$, and let $J_{2}$ denote the intersection of those maximal ideals which are invertible and contain $M$ and not $J$. Since $J_{d}$ is an irredundant intersection of maximal ideals, $J_{d}=J_{1} \cap J_{2}=J_{1} J_{2}$. Since $J_{1}$ is an irredundant intersection of prime trace ideals of $T, J_{1}$ is a trace ideal of $T$ (Houston et al., 2000, Proposition 3.13). Since the only prime ideals of $T$ that contain $J_{2}$ are 
1 invertible maximal ideals of $T, J_{2}$ is an invertible ideal of $T$. We cannot 2 also have $J_{2}\left(S: J_{2}\right)=S$ (unless $\left.S=T\right)$. However, we do have $M J_{2}\left(T: J_{2}\right)=$ $3 M$. It follows that $J_{2}\left(S: J_{2}\right)=M$. Let $t \in(R: I)$. Since $I=J J_{d}=J J_{1} J_{2}$, we 4 have $t J J_{1} \subseteq\left(S: J_{2}\right), \quad t J J_{2} \subseteq\left(J_{1}: J_{1}\right)$ and $t J_{d}=t J_{1} J_{2} \subseteq(J: J)$. Thus $t I \subseteq$ $5 \quad M \cap J \cap J_{1}=J \cap J_{d}=I$. Therefore $I$ is a trace ideal of $S$.

By (a), $\quad J J_{d} \subseteq S$. Hence, $\quad J J_{d}=Q^{\prime} J_{d} \subset Q=Q^{\prime} \cap S$. In the case $J+M \neq T, J \cap S \subseteq M$. Hence $Q=Q^{\prime} \cap S=J \cap S=J \cap M=J \cap J_{d}$.

Theorem 20. For diagram $\square_{4}, S$ is a TPP domain if and only if $T$ is a TPP domain.

Proof. $(\Rightarrow)$ Assume $S$ is a TPP domain and let $Q^{\prime}$ be a $P^{\prime}$-primary ideal of $T$. In any case we have $M^{2} Q^{\prime}\left(T: Q^{\prime}\right) \subseteq Q(S: Q)$.

Case 1. $Q^{\prime}+M=T$.

In this case we also have $Q+M=S$. By checking locally, it is easy to show that $(S: Q) \subseteq\left(T: Q^{\prime}\right)$. If $Q(S: Q)=S$, then $Q^{\prime}\left(T: Q^{\prime}\right)=T$. If $Q(S: Q)=P$, we have $M^{2} Q^{\prime}\left(T: Q^{\prime}\right) \subseteq P=Q(S: Q) \subseteq Q^{\prime}\left(T: Q^{\prime}\right)$. Again by checking locally, we have $Q^{\prime}\left(T: Q^{\prime}\right)=P^{\prime}$.

Case 2. $Q^{\prime}+M \neq T$.

In this case $Q^{\prime} Q^{\prime}{ }_{d}=Q$ and $P^{\prime} Q^{\prime}{ }_{d}=P$. Thus $Q^{\prime} Q^{\prime}{ }_{d}(S: Q)=Q(S: Q)$ $\subseteq S$ and we also have $\left.Q^{\prime} d S: Q\right) \subseteq\left(T: Q^{\prime}\right)$. If $P \neq M$, then $P \subset M$. Thus $P=Q(S: Q)=Q^{\prime} Q_{d}^{\prime}(S: Q) \subseteq Q^{\prime}\left(T: Q^{\prime}\right)$ and $M Q^{\prime}{ }_{d} Q^{\prime}\left(T: Q^{\prime}\right) \subseteq Q(S: Q)=P$. P. Hence $P \subseteq Q^{\prime}\left(T: Q^{\prime}\right) \subseteq P^{\prime}$. As $P T_{N^{\prime}}=P^{\prime} T_{N^{\prime}}$ for each maximal ideal containing $P^{\prime}$, we have $Q^{\prime}\left(T: Q^{\prime}\right)=P^{\prime}$. If $P=M$, then $P^{\prime}$ is a maximal ideal of $T$. It follows that $Q^{\prime}\left(T: Q^{\prime}\right)$ contains $P^{\prime}$ since $T$ is an LTP domain.

$(\Leftarrow)$ Assume $T$ is a TPP domain and let $Q$ be a $P$-primary ideal of $S$ with $P \neq M$. Since $S$ is an LTP domain, we may assume that $P$ is not a maximal ideal of $S$.

Since $P$ is not a maximal ideal of $S, P^{\prime}$ is not a maximal ideal of $T$. Thus $Q^{\prime}\left(T: Q^{\prime}\right)=P^{\prime}$. If $M$ contains $P$, we have $Q(S: Q) \subseteq Q\left(T: Q^{\prime}\right)=$ $Q_{d}^{\prime} Q^{\prime}\left(T: Q^{\prime}\right)=Q_{d}^{\prime} P^{\prime}=P$. Hence $Q(S: Q)=P$. If $M$ does not contain $P$, we at least have $Q+M^{2} P \subseteq Q+M^{2} Q^{\prime}\left(T: Q^{\prime}\right) \subseteq Q(S: Q) \subseteq Q^{\prime}\left(T: Q^{\prime}\right)=P^{\prime}$. $\mathrm{P}^{\prime}$. By checking locally in $S$ we find that $Q(S: Q)=P$.

Theorem 21. For diagram $\square_{4}, S$ is an RTP domain if and only if $T$ is an RTP domain.

Proof. $\quad(\Rightarrow)$ Assume $S$ is an RTP domain and let $J$ be a trace ideal of $T$. 42 Let $I=J J_{d}$. By Lemma 19, $I$ is a trace ideal and for each maximal ideal $N^{\prime}$ 
1 containing $J, I T_{N^{\prime}}=J T_{N^{\prime}}$. Since $S$ is an RTP domain, $I$ is a radical ideal 2 of $S$. Hence by Lemma $19, J$ is a radical ideal of $T$.

$3 \quad(\Leftarrow)$ Assume $T$ is an RTP domain and let $I$ be a trace ideal of $S$. Let $4 J=I(T: I)$. Since $I(S: I)=I$, we have $J M \subseteq I$.

Case 1. $M+J=T$ and $I \subset M$.

In this case $M J=M \cap J=I$. As both $J$ and $M$ are radical ideals of $T$, $I$ is a radical ideal of both $S$ and $T$.

Case 2. $I+M=S$.

For each maximal ideal $N$ containing $I$, we have $J T_{N^{\prime}}=M J T_{N^{\prime}} \subseteq$ $I T_{N^{\prime}}=I S_{N} \subseteq J T_{N^{\prime}}$ where $N^{\prime}$ is the unique maximal ideal of $T$ that contracts to $N$. As $J$ is a radical ideal of $T, I$ is a radical ideal of $S$.

Case 3. $I \subseteq M$ and $M+J \neq T$.

In this case we have $J J_{d}=J \cap J_{d} \subseteq I \subseteq J \cap M=J \cap J_{d}$. Hence $I$ is a radical ideal of both $S$ and $T$.

The next corollary follows from combining the appropriate results above; namely Theorems 12 and 20 and Theorems 13 and 21 .

Corollary 22. For diagram $\square_{5}, R$ is a TPP (RTP) domain if and only if both $T$ and $D$ are TPP (RTP) domains.

In the next section we shall drop the requirement that $M$ be a radical ideal of $T$. Instead we consider the case when the radical of $M$ in $T$ is an invertible ideal of $T$.

Lemma 23. Let $R$ be an LTP domain and let $J$ be an ideal for which each $J_{\alpha}=J R_{M_{\alpha}} \cap R$. If $\sqrt{J}$ is invertible, then the intersection $\bigcap J_{\alpha}$ is irredundant. 
1 Proof. By Lemma 9, $J$ and each ideal that contains $J$ is invertible. As in 2 the proof of Lemma 9, for each $M_{\beta}$ containing $J$, there is an element $3 s \in R \backslash M_{; \beta}$ such that $s J_{; \beta} \subseteq J$. As the ideals $J_{\alpha}$ are incomparable, $s$ is con4 tained in each $J_{\alpha}$ except for $J_{\beta}$. Thus the intersection $\cap J_{\alpha}$ is 5 irredundant.

In our next pullback construction, we no longer assume that $M$ is a radical ideal of $T$. What we will substitute is the assumption that $M$ is an ideal of $T$ whose radical in $T$ is an invertible ideal of $T$. As $M$ is a maximal ideal of $S$, no confusion should arise if we denote the radical of $M$ in $T$ as $\sqrt{M}$. We will continue to have the assumption that each minimal prime of $M$ in $T$ is a maximal ideal of $T$ and that $T / M$ contains a field $F$. With all of these assumptions, let $S$ be the pullback of the following diagram:

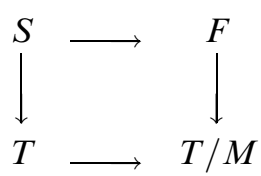

As in diagram $\square_{4}$, we use $M_{\alpha}$ to denote a maximal ideal of $T$ that contains $M$ and use $\mathscr{M}$ to denote the set of such ideals. Since we are no longer assuming $M$ is a radical ideal of $T, M T_{M_{\alpha}}$ need not be equal to $M_{\alpha} T_{M_{\alpha}}$ for each $M_{\alpha}$ in $\mathscr{M}$. We use $Q_{\alpha}$ to denote the $M_{\alpha}$-primary component of $M$; i.e., $Q_{\alpha}=M T_{M_{\alpha}} \cap T$ for each $M_{\alpha} \in \mathscr{M}$. For each ideal $J$ of $T$, we let $J_{b}=\bigcap\left\{Q_{\alpha} \mid J \subseteq M_{\alpha}, M_{\alpha} \in \mathscr{M}\right\}$ and $J_{a}=\bigcap\left\{Q_{\alpha} \mid J \subseteq M_{\alpha}\right.$, $\left.M_{\alpha} \in \mathscr{M}\right\}$.

Theorem 24. For diagram $\square_{6}, S$ is an LTP domain if and only if $T$ is an LTP domain. Moreover, when this is the case, then $M$ is an invertible ideal of $T$.

Proof. As in the proof of Theorem 17, we need only show that a primary ideal can be a trace ideal only if it is prime. Even though we no longer have that $M$ is a radical ideal, the proof given for those cases in Theorem 17 where the radical of the primary ideal does not contain $M$ are valid here. Thus we need only be concerned with those primary ideals which are trace ideals and whose radicals contain $M$.

$\Leftrightarrow$ ) Assume $S$ is an LTP domain and let $Q^{\prime}$ be a primary ideal of $T$ which is also a trace ideal. Let $Q=Q^{\prime} \cap S, P^{\prime}=\sqrt{Q^{\prime}}$ and $P=P^{\prime} \cap S$. As $Q^{\prime}$ is a trace ideal of $T$ so is $P^{\prime}$ (Houston et al., 2000, Proposition 2.1). If $P^{\prime}$ does not contain $M$, repeat the proof given for Cases 1 and $2(\Rightarrow)$ in 
1 Theorem 17 to show that $Q^{\prime}=P^{\prime}$. To complete the proof we will show that $P^{\prime}$ cannot contain $M$.

Since $\sqrt{M}$ is an invertible ideal of $T$, each maximal ideal containing $M$ is locally principal. It follows that $M$ is locally principal as an ideal of $T$ and, therefore, $(S: M)=(M: M)=T$. Assume $P^{\prime}$ contains $M$ and consider the ideal $P^{\prime} M$. As $M$ is locally principal, $P^{\prime} M \neq M$. Since $P^{\prime}$ is a trace ideal of $T$ and $(S: M)=(M: M)=T$, we have $\left(S: P^{\prime} M\right) P^{\prime} M=$ $\left((S: M): P^{\prime}\right) P^{\prime} M=\left(T: P^{\prime}\right) P^{\prime} M=P^{\prime} M$. Thus $P^{\prime} M$ is proper $M$-primary trace ideal of $S$. Since $S$ is an LTP domain, this is impossible. Hence $P^{\prime}$ cannot contain $M$ and $T$ is an LTP domain.

$(\Leftarrow)$ Assume $T$ is an LTP domain and let $Q$ be a primary ideal of $S$ which is also a trace ideal. Let $P$ be the radical of $Q$ (as an ideal of $S$ ). If $P$ is not equal to $M$, repeat the proof given for Case $1(\Leftarrow)$ in Theorem 17 .

Assume $P=M$. By Lemma $9, M$ is an invertible ideal of $T$. Since $Q$ is $M$-primary, $(S: Q)=(Q: Q)$ contains $(S: M)=(M: M)=T$. Thus $Q$ is an ideal of $T$ with the same radical as $M$. Hence $Q$ is an invertible ideal of $T$ and we have $M=M Q(T: Q)$. Therefore, $Q=M$.

Proof. Assume $T$ is an LTP domain. Then by Lemma 9, each ideal in $\mathscr{M}$ is invertible and the intersection $\bigcap_{M_{\alpha} \in \mathscr{M}} M_{\alpha}$ is irredundant. Moreover, by Lemma 23, each $Q_{\alpha}$ is invertible and the intersection $\bigcap_{M_{\alpha} \in M} Q_{\alpha}$ is irredundant. It follows that $J$ and $J_{b}$ are comaximal and that $M=J_{a} J_{b}$. Thus $J \cap J_{b}=J J_{b}$ and $I=J J_{a} J_{b}$. We also have that both $J_{a}$ and $J_{b}$ are invertible.

Since $M$ is an invertible ideal of $T,(S: M)=(M: M)=T$. It follows that $(S: I) I=(S: J M) J M=((S: M): J) J M=(T: J) J M$. Thus $I$ is a trace ideal of $S$ if $J$ is a trace ideal of $T$. 
Assume $J$ is a trace ideal of $T$. Since $J_{a}$ is invertible, if it contains $J$, then $J_{a}(T: J)=(T: J)$ and it follows that $J J_{a}=J J_{a}(T: J)=J(T: J)=J$.

3 As $M=J_{a} \cap J_{b}=J_{a} J_{b}$ we also have $I=J J_{b}=J \cap J_{b}=J \cap M$. To establish

4 (2), all that remains is to show that $J_{a}$ contains $J$. We will do this 5 locally. Let $N^{\prime}$ be a maximal ideal of $T$. If $N^{\prime}$ does not contain $M$, then 6 it cannot contain $J_{a}$. Thus $J T_{N^{\prime}}=J_{a} J T_{N^{\prime}} \subseteq J_{a} T_{N^{\prime}}$. If $N^{\prime}$ contains $M$ and 7 does not contain $J$, then both $J T_{N^{\prime}}$ and $J_{a} T_{N^{\prime}}$ are equal to $T_{N^{\prime}}$. If $N^{\prime}$ 8 contains both $M$ and $J$, then $N^{\prime}$ is invertible and $(T: J)$ contains $9 \quad\left(T: N^{\prime}\right)$. It follows that $N^{\prime}(T: J)=(T: J)$ and, therefore, $10 N^{\prime} J=N^{\prime} J(T: J)=J(T: J)=J$. As $J_{a} T_{N^{\prime}}=N^{\prime k} T_{N^{\prime}}$ for some positive integer $k$, we have $J T_{N^{\prime}}=N^{\prime k} J T_{N^{\prime}}=J_{a} J T_{N^{\prime}} \subset J_{a} T_{N^{\prime}}$. Therefore $J_{a}$ contains $J$ and the proof of (2) is complete.

For (3), let $Q^{\prime}$ be a primary ideal of $T$ with radical $P^{\prime}$ and assume $P^{\prime}$ is neither maximal nor comaximal with $M$. Let $M_{\alpha}$ be a maximal ideal that contains both $P^{\prime}$ and $M$. Since $M_{\alpha}$ is invertible, there is an element $r \in M_{\alpha}$ such that $r T_{M_{\alpha}}=M_{\alpha} T_{M_{\alpha}}$. Let $p$ be an element of $Q^{\prime}$. Then there is an element $s \in T_{M_{r}}$ such that $p=s r$. As $Q^{\prime}$ is $P^{\prime}$-primary and $r$ is not in $P^{\prime}$, $s$ must be in $Q^{\prime} T_{M_{\alpha}}$. It follows that $Q_{a}^{\prime} Q^{\prime} T_{M_{\alpha}}=Q^{\prime} T_{M_{\alpha}}$. Thus $Q^{\prime} Q^{\prime}{ }_{a}=Q^{\prime}$.

Since $P^{\prime}$ and $M$ are not comaximal, $Q^{\prime} \cap S=Q^{\prime} \cap M=Q^{\prime} \cap Q^{\prime}{ }_{a} \cap Q_{b}^{\prime}$. As $Q^{\prime} Q^{\prime}{ }_{a}=Q^{\prime}$ and $Q^{\prime}+Q^{\prime}{ }_{b}=T, Q^{\prime} \cap Q^{\prime}{ }_{a}=Q^{\prime}$ and $Q^{\prime} \cap Q^{\prime}{ }_{b}=Q^{\prime} Q^{\prime}{ }_{b}$. Thus $Q^{\prime} \cap S=Q^{\prime} Q^{\prime}$.

Theorem 26. For diagram $\square_{6}$, S is a TPP domain if and only if $T$ is a TPP domain.

Proof. $(\Rightarrow)$ Assume $S$ is a TPP domain and let $Q^{\prime}$ be a primary ideal of $T$. Let $Q=Q^{\prime} \cap S, P^{\prime}=\sqrt{Q^{\prime}}$ and $P=P^{\prime} \cap S$. Since $Q^{\prime}$ is $P^{\prime}$-primary, the ideals $Q_{b}^{\prime}$ and $P_{b}^{\prime}$ coincide as do the ideals $Q_{a}^{\prime}$ and $P_{a}^{\prime}$. Since $S$ is also an LTP domain, $T$ is an LTP domain. Thus we at least have $P^{\prime} T_{P^{\prime}} \subseteq Q^{\prime}\left(T: Q^{\prime}\right) T_{P^{\prime}}$. If $P^{\prime}$ is maximal, this is all we need to show. Hence we may assume $P^{\prime}$ is not maximal. It follows that $P^{\prime} T_{P^{\prime}}=Q^{\prime}\left(T: Q^{\prime}\right) T_{P^{\prime}}$ and $P^{\prime}$ is a trace ideal of $T$ so $P^{\prime} P_{a}^{\prime}=P^{\prime}$ and $P^{\prime} \cap M=P^{\prime} P_{b}^{\prime}$. We also have that $P$ is a trace ideal of $S$ and $Q(S: Q)=P$. Thus $M^{2} Q^{\prime}\left(T: Q^{\prime}\right) \subseteq$ $Q(S: Q)=P$ and $M Q(S: Q)=M P \subseteq Q^{\prime}\left(T: Q^{\prime}\right)$. If $M$ and $P^{\prime}$ are comaximal, $M P T_{N^{\prime}}=P T_{N^{\prime}}=P^{\prime} T_{N^{\prime}}$ for each maximal ideal $N^{\prime}$ containing $P^{\prime}$ and, therefore, $Q^{\prime}\left(T: Q^{\prime}\right)=P^{\prime}$. If $M$ and $P^{\prime}$ are not comaximal, then $P=P^{\prime} \cap M=P^{\prime} P_{b}^{\prime}=P^{\prime} M$ and $Q=Q^{\prime} \cap M=Q^{\prime} P^{\prime}{ }_{b}$. It follows that $M P=P^{\prime} P_{b}^{\prime 2} \subseteq Q\left(T: Q^{\prime}\right)$. Checking locally we find $P^{\prime}=Q^{\prime}\left(T: Q^{\prime}\right)$ since $Q^{\prime}$ and $P_{b}^{\prime}$ are comaximal.

$(\Leftarrow)$ Assume $T$ is a TPP domain and let $Q$ be a $P$-primary ideal of $S$. If $P=M$, then $Q T$ is an invertible ideal of $T$. Hence we have $M=M Q(T: Q)$. It follows that $Q(S: Q)$ contains $M$. 
If $P \neq M$, then there is a unique prime ideal $P^{\prime}$ of $T$ that contracts to $P$ and a unique $P^{\prime}$-primary ideal $Q^{\prime}$ that contracts to $Q$. We again have $M^{2} Q^{\prime}\left(T: Q^{\prime}\right) \subseteq Q(S: Q)$ and $M(S: Q) \subseteq\left(T: Q^{\prime}\right)$. Since $S$ is an LTP 4 domain, if $P$ is a maximal ideal of $S$, we will have $P \subseteq Q(S: Q)$. Thus we can assume $P$ is not maximal. Since $T$ is a TPP domain, we have $Q^{\prime}\left(T: Q^{\prime}\right)=P^{\prime}$ so $Q(S: Q)$ contains $M^{2} P^{\prime}=P^{\prime} P_{b}^{\prime 2}$. If $P$ and $M$ are comaximal, we obtain the desired conclusion that $Q(S: Q)=P$ by checking locally in $S$. If $M$ contains $P$, then we have $P=P^{\prime} P^{\prime}{ }_{b}=P^{\prime} M$ and $Q=P_{b}^{\prime} Q^{\prime}=Q^{\prime} M \quad$ by Lemma 25 . Hence $Q(S: Q) \subseteq Q\left(T: Q^{\prime}\right)=$ $P_{b}^{\prime} Q^{\prime}\left(T ; Q^{\prime}\right)=P_{b}^{\prime} P^{\prime}=P$.

Theorem 27. For diagram $\square_{6}, S$ is an RTP domain if and only if $T$ is an RTP domain.

Proof. $(\Rightarrow)$ Assume $S$ is an RTP domain and let $J$ be a trace ideal of $T$. Then $\sqrt{J}$ is also a trace ideal of $T$ (Houston et al., 2000, Proposition 2.1) and $T$ is an LTP domain. Let $I=J M$ and $C=\sqrt{J} M$. By Lemma 25, $I=J J_{b}, C=\sqrt{J} J_{b}$ and both are a trace ideals of $S$. Since $S$ is an RTP domain, both $I$ and $C$ are radical ideals of $S$. It follows that $I=C$ so $J J_{b}=\sqrt{J} J_{b}$. Since no maximal ideal of $T$ can contain both $J$ and $J_{b}$, we find that $J=\sqrt{J}$ by checking locally.

$(\Leftarrow)$ Assume $T$ is an RTP domain and let $I$ be a trace ideal of $S$. Let $J=I(T: I)$. Since $I(S: I)=I$, we have $J M \subseteq I$. By Lemma $25, J M=J \cap M$. Since $T$ is an RTP domain, $J$ is a radical ideal of $T$. Thus $J \cap S$ is a radical ideal of $S$. If $I$ and $M$ are comaximal, we find that $I=J \cap S$ by checking locally in $S$. If $I$ and $M$ are not comaximal, then $M$ contains $I$ and we have $J \cap M=J M \subseteq I \subseteq J \cap M$. Thus in either case, $I$ is a radical ideal of $S$. Therefore, $S$ is an RTP domain.

For diagram $\square_{6}$, let $D$ be a domain contained in $F$ and let $R$ be the pullback of the following diagram:

Combining Theorem 27 with Theorems 11, 12 and 13 we have the following.

Corollary 28. For diagram $\square_{7}, R$ is an LTP (TPP) [RTP] domain if and

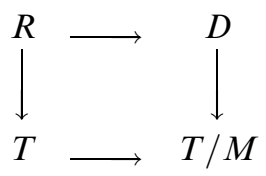


Theorem 30. For diagram $\square_{7}$, assume further that $T$ is a Dedekind domain. Then $R$ is a TP domain if and only if $D$ is a TP domain.

Proof. Let $P$ be a (nonzero) prime ideal of $R$ other than $M$. Then $P$ is either the contraction of a maximal ideal $N^{\prime}$ of $T$ or $P$ is the inverse image of a nonzero prime $\bar{P}$ of $D$. If $P=N^{\prime} \cap R$, then it is invertible as an ideal of $R$. If $P$ is the inverse image of some prime $\bar{P}$ of $D$, then $P$ is invertible if and only if $\bar{P}$ is invertible (Fontana and Gabelli, 1996, Corollary 1.7).

Now, if $R$ is a TP domain, then $R / P$ is a TP domain for each prime ideal $P$ (Cahen and Lucas, 1997, Corollary 11). Thus $R$ is a TP domain only if $D$ is a TP domain. Conversely, if $D$ is a TP domain, then the noninvertible prime ideals of $D$ are linearly ordered. It follows that the noninvertible prime ideals of $R$ are linearly ordered. The conclusion follows from Corollary 29 and the fact that a domain is a TP domain if and only if it is an RTP domain for which the noninvertible primes are linearly ordered (Cahen and Lucas, 1997, Corollary 11).

\section{EXAMPLES}

We conclude with three examples. In the first two, we show that $T$ can have a trace property while $S$ does not when we only have that $M$, and not the radical of $M$ in $T$, is invertible as an ideal of $T$ even if the radical of $M$ in $T$ is a maximal ideal. In the first of these, $T$ is a Noetherian domain whose integral closure is a PID. In the second, $T$ is onedimensional valuation domain which is not Noetherian. The third is the one promised with regard to TP domains and diagram $\square_{1}$.

Example 31. Let $T=F\left[x^{2}, x^{3}\right]$ and $S=F\left[x^{2}, x^{5}\right]$ with $M=\left(x^{2}, x^{5}\right) S$. Then $T$ is an RTP domain and $M=X^{2} T$ is an invertible ideal of $T$, but the radical of $M$ in $T$ is the maximal ideal $N=\left(x^{2}, X^{3}\right) T$ which is 
1 not invertible (as an ideal of $T$, but is invertible in $F[x]=(T: N)$ ). The $2 \operatorname{ring} S$ is not even an LTP domain. The ideal $I=\left(x^{4}, x^{5}\right) S$ is a proper $3 M$-primary trace ideal of $S$.

Example 32. Let $T$ be a one-dimensional valuation ring of the form $F+N$ which is not discrete and let $x$ be a nonzero nonunit of $T$. Let $M=x T$ and $S=F+M$. Since $T$ is a valuation domain, it has the trace property. Obviously, $M$ is an invertible ideal of $T$, but its radical is not. The ideal $I=x N$ is a proper $M$-primary trace ideal of $S$. Thus $S$ is not even an LTP domain.

Example 33. Let $F$ be a field and let $X$ and $Y$ be indeterminates over $F$. Set $T=F[Y]+X F(Y)[X], M=(X+1) F(Y)[X] \cap T$ and $Q=X F(Y)[X]$. Let $R$ be the pullback in the following diagram:

Then

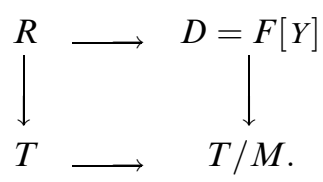

Proof. Since $D=F[Y]$ is a PID, it is a TP domain. For $T$, first note that $Q$ is a common prime ideal of $T$ and $F(Y)[X]$. Thus, as both $F[Y]$ and $F(Y)[X]$ are PIDs, $T$ is a TP domain by Theorem 30 . We also have that $M=(X+1) T($ Costa et al., 1978, Theorem 4.21), so it is an invertible maximal ideal of $T$. Therefore, $(M: M)=T$. As $Q+M=T$, we have that $T / M=F(Y)[X] /(X+1)=F(Y)$ and that $J=Q M$. Now $(R: M)=(M: M)=$ $T$ by (Houston et al. (2000, Corollary 3). Similarly, $(T: Q)=(Q: Q)=$ $F(Y)[X] . \quad$ It follows that $(R: J)=(R: Q M)=((R: M): Q)=(T: Q)=$ $(Q: Q) \subseteq(Q M: Q M)=(J: J)$. So $J$ is a trace ideal of $R$. But, obviously, $J$ is not a prime ideal of $R$. Hence $R$ is not a TP domain.

\section{ACKNOWLEDGMENT}

40

During the preparation of this paper, Professor Kabbaj was supported by KFUPM. 
Anderson, D. F. (1983). When the dual of an ideal is a ring. Houston J. Math. 9:325-332.

Anderson, D. F., Dobbs, D. E. (1980). Pairs of rings with the same prime ideals. Canad. J. Math. 32:362-384.

Anderson, D. D., Huckaba, J., Papick, I. (1987). A note on stable domains. Houston J. Math. 13:13-17.

Bass, H. (1963). On the ubiquity of Gorenstein rings. Math. Z. 82:8-28.

Barucci, V. (1986). Strongly divisorial ideals and complete integral closure of an integral domain. J. Algebra 99:132-142.

Cahen, P.-J., Lucas, T. (1997). The special trace property, Commutative Ring Theory (Féz, 1995). Lecture Notes in Pure and Appl. Math., Vol. 185. New York: Dekker, pp. 161-172.

Costa, D., Mott, J., Zafrullah, M. (1978). The construction $D+x D_{S}[x]$. J. Algebra 53:423-439.

Fontana, M. (1973). Topologically defined classes of commutative rings. Ann. Math. Pura Appl. 123:331-355.

Fontana, M., Gabelli, S. (1996). On the class group and the local class group of a pullback. J. Algebra 181:803-835.

Fontana, M., Huckaba, J., Papick, I. (1987). Domains satisfying the trace property. J. Algebra 107:169-182.

Fossum, R. (1973). The Divisor Class Group of a Krull Domain. New York: Springer-Verlag.

Gabelli, S. (1992). Domains with the radical trace property and their complete integral closure. Comm. Algebra 20:829-845.

Gilmer, R. (1972). Multiplicative Ideal Theory. New York: Dekker.

Hedstrom, J., Houston, E. (1978). Pseudo-valuation domains. Pacific J. Math. 75:137-147.

Heinzer, W., Papick, I. The radical trace property. J. Algebra 112: $110-121$.

Houston, E., Kabbaj, S., Lucas, T., Mimouni, A. (1995). Duals of ideals in pullback constructions. Zero-Dimensional Commutative Rings (Knoxville, 1994), Lecture Notes in Pure and Appl. Math. Vol. 171. New York: Dekker, pp. 263-276.

Houston, E., Kabbaj, S., Lucas, T., Mimouni, A. (2000). When is the dual of an ideal a ring?. J. Algebra 225:429-450.

Huckaba, J., Papick, I. (1982). When the dual of an ideal is a ring. Manuscripta Math. 37:67-85.

Kabbaj, S., Lucas, T., Mimouni, A. 1999. Trace Properties and integral domains, Advances in Commutative Ring Theory (Fés, 1997), 


\section{3_AGB31_03_R1_121902}

Trace Properties and Pullbacks

1 Lecture Notes in Pure and Appl. Math. Vol. 205. New York: ED1 2 Dekker, pp. 421-436.

3 Lucas, T. (1996). The radical trace property and primary ideals. 4 J. Algebra 184:1093-1112. 